\title{
LITERATURA SICILIANA CONTEMPORÁNEA: LA IRRUPCIÓN DE LAS MUJERES EN EL PARADIGMA LITERARIO INSULAR ${ }^{1}$
}

\author{
José García Fernández²
}

\section{Literatura siciliana contemporánea: la irrupción de las mujeres en el paradigma literario insular}

Resumen: Existen en la bibliografía especializada abundantes estudios literarios sobre escritores sicilianos ligados al pasado. Sin embargo, la acuciante escasez de investigaciones centradas en el nuevo milenio -y, más en concreto, en las mujeres de esta centuria- deja al descubierto un vasto territorio aún por explorar desde el rigor y la exhaustividad que se merecen. Este artículo profundiza en el estudio de la "sicilianidad" desde una perspectiva de género, presenta en su contexto a las principales autoras de la literatura siciliana contemporánea, $\mathrm{y}$ establece los temas y preocupaciones que jalonan la andadura escritural de estas literatas isleñas.

Palabras clave: Literatura italiana, cultura siciliana, escritoras sicilianas contemporáneas, cambio de paradigma literario, autoras y género.

Contemporary Sicilian Literature: the Upsurge of Women Writers in the Literary Paradigm of the Island

Abstract: Literary studies about Sicilian writers related to the past abound in the specialized bibliography. However, the abysmal lack of research centred upon the new millenium -and most especially, on women writers of this century- unveils a vast territory yet to explore with the rigour and exhaustiveness it requires. This article delves into the study of 'Sicilianness' from a gender perspective, discusses in context the main women writers of contemporary Sicilian literature, and sets up the themes and concerns that mark the literary paths of these female authors.

Key words: Italian literature, Sicilian culture, contemporary Sicilian women writers, change of literary paradigm, authors and gender.

\section{Introducción}

A resultas del patriarcado y de la religiosidad isleñas, las sicilianas siempre han sido minusvaloradas por un colectivo masculino que, para conseguir doblegarlas, ha

\footnotetext{
${ }^{1}$ Fecha de recepción: 04/04/2019.

Fecha de aceptación: 28/11/2019.

${ }^{2}$ Docente e Investigador, Departamento de Filología Clásica y Románica, Área de Filología Italiana, Universidad de Oviedo, España; $\square$ garciafernandezjose@ uniovi.es. El autor es beneficiario de una ayuda postdoctoral del Programa "Severo Ochoa" para la formación en investigación y docencia del Principado de Asturias (referencia: PA-17-PF-BP16053).
} 
fomentado su analfabetismo ${ }^{3}$. El mero cuestionamiento de las competencias asignadas por tradición las colocaba en un mundo paralelo, marginal y temerario del que no todas salían victoriosas. Interesadas en adquirir independencia y en dar cuenta de su valía profesional, muchas de ellas fueron vilipendiadas por su "osadía". Otras, más afortunadas, lograron su objetivo e iniciaron su propia carrera laboral, si bien, atendiendo exclusivamente a razones de género, nunca alcanzaron -en consonancia con el resto de Italia- la paridad en el reconocimiento de sus competencias (Pruna 2010; Casarico y Profeta 2011). Piénsese, por ejemplo, en la remuneración salarial de las sicilianas, depreciada durante años pese a desempeñar el mismo cargo que sus homólogos varones ${ }^{4}$. En conexión con esta infravaloración femenina, M. González de Sande apunta:

En la sociedad italiana, la mujer era considerada como un complemento del hombre, $y$, aunque fuera necesaria para el mantenimiento del género humano, función primordial de esta, se la estimaba incapaz de gozar de autonomía a consecuencia de su inferioridad natural con respecto al sexo opuesto. Siguiendo estas premisas, muchos de los "ilustres" pensadores de la época [el s. XIX], de la talla de Vincenzo Gioberti o Antonio Rosmini, al igual que harán otros en el resto de Europa, lanzaban sus teorías acerca de la subordinación de la mujer. [...] Teorías todas, sostenidas, desgraciadamente, por una buena parte de la población italiana y que seguirán sentando las bases del derecho de familia hasta que este fue reformado en 1975. (González de Sande y García Fernández 2018: 12-13)

Ante este subyugante panorama, no es de extrañar que muy pocas mujeres se atreviesen a ejercer -al menos públicamente- de escritoras. A sabiendas de que serían repudiadas por no atender en exclusiva su hogar, hallaban en su fascinación por el ámbito literario un doble problema: por un lado, se criticaba su afán por desempeñar un

\footnotetext{
${ }^{3} \mathrm{~A}$ tal respecto, téngase en cuenta que "i tassi di analfabetismo tra le donne andavano, ancora nel 1871, dal 51 per cento del Piemonte al 95 per cento in Basilicata e Calabria e al 91-92 per cento in Sicilia e Sardegna" (D’Amico 2015: 190). El resaltado en negrita es mío.

${ }^{4}$ Esto explica por qué, especialmente en el ámbito agrario, muchos hombres y trabajadores "stentano ad accettare l'uguaglianza dei diritti della donna lavoratrice ed il principio che la donna a parità di lavoro deve essere pagata come loro" (Mafai et al. 1976: 125).
} 
trabajo que las dotase de una libertad de la que a priori solo podían gozar sus padres, hermanos o maridos; por otro, se les reprochaba su atracción por un mundo creativo del que se tenía una imagen perniciosa y distorsionada (Rasy 1984; Briganti 1989; Corona 1990; Pigozzi y De Petris 1998; Iuso 1999; Woolf 2003; Bellerio 2007; Cazalé Bérard 2009).

De todos modos, este fenómeno no solo se circunscribió al área siciliana, sino que también engarzó de manera natural con las demandas sociales de un colectivo mundial que encontró en las reclamaciones y en las críticas de Virginia Woolf a una de sus grandes pensadoras ${ }^{5}$. Así pues, abanderada del feminismo y símbolo destacado de la literatura universal, esta mujer pionera y adelantada a su tiempo no tuvo reparo en afirmar cuanto sigue:

Qualunque effetto lo scoraggiamento e le critiche ebbero sulla loro scrittura [delle donne] - e credo che ebbero un effetto straordinario - questo fu poco importante a confronto con l'altra difficoltà che dovettero affrontare (stavo ancora considerando quelle scrittrici del primo Ottocento) quando decisero di affidare alla carta i loro pensieri: il fatto cioè che non avessero alcuna tradizione alle loro spalle, o che questa fosse talmente breve e parziale da essere di poco aiuto. Perché se siamo donne, riflettiamo sul passato attraverso le nostre madri. È inutile chiedere aiuto ai grandi scrittori, per quanto piacere ci possano procurare. (Woolf 1995: 97)

De acuerdo con estas concepciones, el enmascaramiento de la producción literaria de las escritoras es un hecho difícil de refutar, de ahí que se haga necesario realizar un estudio que dé visibilidad, en este caso concreto, a las autoras sicilianas contemporáneas que con sus textos han conseguido afianzar -dentro de una sociedad moderna que poco a poco avanza hacia la igualdad- su rol de mujer y han logrado reconocer el esfuerzo de sus antecesoras.

\footnotetext{
${ }^{5}$ A ella se suman figuras tan relevantes como Jane Austen, Emily Dickinson, Caterina Percoto, Katherine Mansfield, Anaïs Nin, Anna Banti, Karen Blixen, Dolores Prato o Marguerite Youcenar (Livi 1992, 2002). Asimismo, son igualmente dignas de consideración pensadoras de la talla de Sylvia Plath, Marguerite Duras, Marina Cvetaeva, Ingeborg Bachmann y María Zambrano (Tommasi 2004).
} 


\section{EI nuevo milenio como cambio en el paradigma literario siciliano}

A caballo entre la segunda mitad del siglo XX y los albores del nuevo milenio, muchos intelectuales sicilianos han sido partícipes de los enormes cambios sociales que se han producido en el mundo a lo largo de las últimas décadas. Y si bien, en comparación con autores sicilianos precedentes (Sciascia, Pirandello, etc.), buena parte de estos escritores todavía son desconocidos -piénsese en literatos como Carmelo Freni, Emilio Isgrò, Giovanni Occhipinti, Giuseppe Risica, Lucio Zinna, Matteo Collura, Nino Famà y Sebastiano Saglimbeni ${ }^{6}$, no menos significativo resulta comprobar hasta qué punto el ámbito literario siciliano siempre ha estado en manos de los hombres ${ }^{7}$. Su hegemonía ha puesto de manifiesto el sometimiento que históricamente han sufrido las mujeres isleñas, quienes, con tesón, lograron ir ampliando sus derechos a finales del siglo pasado (Calapso 1980), encontrando en el nuevo milenio, como bien apunta C. Aliberti, un punto de inflexión en sus carreras:

Nel passato l'uomo ha confinato la sua "dolce metà" solo tra le coltri e anche i teologi hanno quasi sempre sostenuto la supremazia del maschilismo, rifacendosi alla testimonianza della Bibbia, addirittura fin dal peccato originale. [...] Le donne, nel corso dei secoli, hanno lasciato rarissime testimonianze nel campo delle lettere, anzi, in testi di diverse epoche, sono apparse eroine negative, simboli di tendenze pervertitrici e causa di guerre e calamità di ogni genere (si pensi, oltre ad Eva, a Elena, a Didone). Per secoli, dunque, la donna è stata relegata nell'ambito dell'esercizio del potere creativo solo nella sfera biologica. [...] [Tuttavia] dall'ultimo dopoguerra, con l'avvento della democrazia, sulla spinta di movimenti protestatari, nell'ultimo trentennio l'inserimento della donna nel mondo letterario e poetico ha determinato un vero e proprio fenomeno sociale in quanto donne

\footnotetext{
${ }^{6}$ Nótese, no obstante, que, tal y como señala Aliberti (2008: 478-479), el autor que realmente ha logrado consolidarse dentro de este innovador panorama literario ha sido Andrea Camilleri, un escritor que ha sabido innovar y reinventar el horizonte siciliano hasta el punto de convertir sus obras en un fenómeno de masas. Así pues, continúa Aliberti (2008: 480), no es de sorprender que Camilleri haya sabido jugar con el estilo artístico de sus composiciones, atendiendo para ello a múltiples elementos narrativos que, por un lado, han hecho de su producción una de las más representativas del humanismo ilustrado italiano y que, por otro, han permitido reinterpretar con acierto la compleja historia de Sicilia.

7 Basta con consultar algunos de los principales manuales de literatura siciliana contemporánea para percatarse de ello (Ferlita 2004; Aliberti 2008, 2013).
} 
scrittrici, poetesse, saggiste dominano tante pagine della nostra letteratura. (Aliberti 2008: 685, 688)

Así pues, a la vasta lista de escritores e intelectuales sicilianos, se ha ido sumando un número cada vez mayor de autoras, literatas que han sabido dar voz a sus legítimas aspiraciones personales y colectivas (Baldaro Verde y Nappi 2002), que se han rebelado contra el opresivo arquetipo social impuesto y que han introducido con habilidad en sus composiciones nuevos puntos de vista sobre un territorio que pareciera haberse olvidado de ellas.

A este respecto, G. Resta afirma que "da Pirandello in poi [...] la 'presenza femminile' assume una diversa dimensione” (Resta 1984: 8), de ahí que la importancia de las mujeres en tanto que procreadoras de la especie, el análisis crítico de su rol hogareño y familiar o la reivindicación de una necesaria igualdad de oportunidades hayan pasado a constituir aspectos clave de la "sicilianidad" (Randazzo 1985). De entre las múltiples mujeres que se han hecho un hueco en el panorama literario insular actual sobresalen con todo merecimiento dos grandes poetas: Jolanda Insana ${ }^{8}$ y Mirella Genovese, dos intelectuales que han sabido adentrarse en un nuevo paradigma cultural que, aun manteniendo la fascinación por los mitos y las tradiciones, ha provocado una inversión en la tendencia escritural de la isla. En relación con Insana, cabe indicarse cuanto sigue:

\begin{abstract}
Nell'universo della poetessa, in cui è assente il Dio rivelato, la poesia ha rappresentato lo strumento lieve di relazione tra il mondo invaso dal male e il catartico respiro dell'Eterno ignoto. Gli strumenti linguistici risultano sempre in sintonia con la valenza delle scelte tematiche. [...] [La Insana] inserisce la poesia nella babele dei linguaggi, dove il "pastiche" linguistico diventa una realtà, in cui convivono la compresenza dell'allegoria, dell'ironia, della parodia, la fusione del linguaggio letterario con quello mediale, ma anche la denuncia delle mistificazioni del linguaggio televisivo, assunto a sistema mistificatorio del vuoto e della gratuita violenza di cui si fa portavoce. (Aliberti 2008: 692, 700)
\end{abstract}

\footnotetext{
${ }^{8}$ La editorial Garzanti publicó en 2007 un libro titulado Tutte le poesie 1977-2006 en el que aparecen recogidos la mayoría de los poemas escritos por esta autora.
} 
Haciendo gala de un portentoso virtuosismo estilístico y pendiente de las profundas transformaciones en el devenir social de Italia y del mundo, Insana volcó todos sus conocimientos en la poesía y comenzó su carrera lírica en 1977 con su obra Sciarra amara, volumen al que siguieron Fendenti fonici (1982), Il collettame (1985), La clausura (1987), Medicina carnale (1994), L'occhio dormiente (1997), La stortura (2002), La tagliola del disamore (2005), Satura di cartuscelle (2008), Frammenti di un oratorio per il centenario del terremoto di Messina (2009), Turbativa d'incanto (2012) y Cronologia delle lesioni (póstuma, 20179). Sin embargo, más allá de esto, ha de notarse cómo la característica más singular de sus escritos, donde deja patente el dolor y el sufrimiento físico de la especie humana, es su impronta siciliana, particularidad, por otra parte, innegable, si se tiene en cuenta que "la poetessa ha ereditato dallo scrigno della sua isola quel patrimonio di valori, connotativi della sua gente, arricchiti attraverso l'itinerario dei suoi studi e sintetizzabili nel credo assoluto della libertà" (Aliberti 2008: 690).

De todas formas, Mirella Genovese ha dado igualmente testimonio de su estrecho vínculo con Sicilia a través de su producción literaria:

La poesia della Genovese, paradigmata in versi liberi, è soffusa di una sommessa musicalità e utilizza un lessico concreto, desunto dal paesaggio della sua terra e da elementi della natura che palpitano di inespresse emozioni, di contenuti moti dell'anima, di figurazioni anatomiche della fisicità, lapidariamente delineate, senza apparente cedimento al pulsare del sentimento. (Aliberti 2008: 702)

Inspirándose en el paisaje de su tierra, Genovese pone de manifiesto el influjo que sobre ella ejerce el territorio insular, logrando, de este modo, mostrar no solo un bagaje

\footnotetext{
${ }^{9}$ Téngase presente que Jolanda Insana falleció en 2016. 
personal en el que su sólida formación académica y su ligazón con Sicilia se tornan rasgos esenciales de su personalidad, sino además un insigne interés por una región que también queda representada en sus composiciones ${ }^{10}$. Y si bien la relevancia de esta autora -en consonancia con numerosas literatas insulares- es digna de alabar, son muchas las escritoras sicilianas, sobre todo de género narrativo, las que han sabido dar cuenta de sus capacidades y aptitudes en los últimos años.

Entre las autoras más conocidas a nivel internacional se encuentran Teresa Carpinteri, Maria Rosa Cutrufelli, Laura Di Falco, Silvana Grasso, Simonetta Agnello Hornby, Giuseppina Torregrossa y Livia De Stefani. En cambio, entre las menos estudiadas se hallan Marinella Fiume, Simona Lo Iacono, Anna Mallamo, Nadia Terranova, Veronica Tomassini, Lina Maria Ugolini, Emma Dante, Licia Cardillo Di Prima, Daniela Gambino, Cleria Lombardo, Beatrice Monroy, Marilena Monti y Clara Serretta. No obstante, siguiendo una visión integradora, se ha considerado útil la realización de un análisis comparativo centrado en los temas y en los aspectos que cada una de estas escritoras aborda en sus obras, conscientes de que solo así será posible: 1) determinar cuáles son los principales puntos de unión (semejanzas) entre estas literatas; y 2) esclarecer cuáles son las características más representativas de su producción literaria.

Para alcanzar estos objetivos, como se ha venido haciendo hasta el momento, se usarán, además de los múltiples libros publicados por las autoras sicilianas mencionadas -haciéndose igualmente acopio de los datos que la mayoría de ellas ofrece en sus páginas webs personales-, los principales manuales y monográficos centrados en la literatura siciliana contemporánea.

\footnotetext{
${ }^{10}$ Algunas de sus obras más significativas son Codice segreto (1999), Pupattola (2003) -escrito junto a Mirellina Paesi-, Cartografia (2004) y Ascolto (2006).
} 


\section{Temáticas y lugares comunes en las escritoras sicilianas contemporáneas}

Buena parte de las intelectuales sicilianas más recientes -incluyéndose a Anna Mallamo, de origen calabrés, y a Nadia Terranova, atenta tanto a Sicilia ${ }^{11}$ como al universo eslavo- muestra un notable interés por su zona de origen, un territorio del que no siempre se sienten satisfechas. Conscientes de las trabas y de los obstáculos a los que tienen que enfrentarse las mujeres isleñas, brindan al lector una visión homogénea de una región que no aprecian por igual, pero sobre la que comparten la misma opinión. A su juicio, Sicilia es un lugar dominado por el palpable machismo -también perceptible, tal y como Daniela Gambino pone de manifiesto, en la misoginia y en la homofobia ${ }^{12}-$, un lugar donde las mujeres desempeñan un papel secundario que dificulta su visibilidad (Correnti 2001; Letizia 2008; Lo Jacono y Zanda 2013).

Aun así, Marilena Monti reconoce estar enamorada del dialecto, la cultura y la historia insulares ${ }^{13}$, un parecer que choca de lleno con el sufrimiento padecido por Livia

11 En concreto, a Mesina, su ciudad natal, a la que hace referencia en su producción literaria, manifestando la fuerte conexión que siente con su localidad de origen. Esto explica por qué "la Messina di Terranova è una città in formazione, mai diventata metropoli, anche urbanisticamente distante dalle grandi città sia del Sud che del Nord. [...] Nel caso di Nadia Terranova, Messina è il luogo che condanna la perifericità, il punto della Sicilia più proteso verso il resto dell'Europa, ma paradossalmente il più difficile da lasciare" (Savatteri 2017) -el libro carece de paginación en el formato electrónico-.

12 Téngase en cuenta que Daniela Gambino ha publicado varias obras -tanto ensayos como novelasrelacionadas con la misoginia y la homofobia imperantes en la sociedad italiana (y de manera destacada en la siciliana); con la situación del feminismo actual y la inserción de las mujeres en este nuevo contexto; con los malos hábitos adquiridos, el autodescubrimiento y la felicidad humana; y con las tradiciones, las historias, las leyendas, los atractivos y los lugares emblemáticos de un complejo territorio, el siciliano, en el que por desgracia la mafia ha jugado -y sigue jugando- un papel esencial.

13 Tanto es así que esta autora ha sabido verter con maestría y de forma crítica su pasión por Sicilia en las obras publicadas hasta la fecha: Giudice Paolo (1993), Foulard (2000), Passione (2000), Viaggio di cuore (2000), Gabriele (2002), L'Isola Signora. Emozioni in Sicilia (2005), I «pizzini» della legalità (2006), Stupore irriverente. L'ultimo scritto di Salvatore Giuliano (2006), Il rito del tè (2013) e Isola Emozione (2016). 
De Stefani: empeñada en dedicarse a la literatura pese a la oposición de sus parientes, el hermetismo ideológico de su familia acabó por hacerla esclava de los estándares de una población que vivía atrapada en el pasado ${ }^{14}$. No es de extrañar, por tanto, que su retrato de la "sicilianidad" adquiera un aspecto reivindicativo e implacable, pues solo así se podían sacar a la luz las atrocidades de un área anclada en sus tradiciones. A tal respecto, cabe señalar:

In Sicilia, dove sempre vivo era stato il culto geloso delle proprie memorie, gli studi delle tradizioni popolari ebbero numerosi e insigni cultori, come S. Guastella, Corrado Avolio e G. Schirò. Ma chi sottrasse veramente questi studi alla frammentarietà e alla dispersa mania di curiosità, trattandoli con rigore e metodo scientifico, fu Giuseppe Pitrè da Palermo (1841-1916), che fu medico e garibaldino e infine docente di Demopsicologia all'Università di Palermo. A lui si deve il Museo etnografico siciliano di Palermo. Scrisse numerose opere, la più importante delle quali è la "Biblioteca delle tradizioni popolari siciliane", in 25 volumi. Con il Pitrè vanno ricordati Gaetano Di Giovanni e Salvatore Salomone-Marino, che con lui collaborarono attivamente e quegli studi illustrarono con importanti opere. (Cucinotta 1996: 202-203)

Seducidas, en definitiva, por las costumbres sicilianas, Licia Cardillo Di Prima, Teresa Carpinteri, Laura Di Falco, Maria Rosa Cutrufelli, Silvana Grasso, Daniela Gambino, Beatrice Monroy y Marilena Monti también reflexionan acerca de su tierra natal, un espacio cuyas singularidades culturales -hábitos, historia, religión, conservadurismo, mafia, extorsión, rencor, abyección colectiva, opresión sexual, mitos, familia, gastronomía, arte- describen con esmero y gran profusión. De entre estas particularidades, la emigración es, quizá, el punto más significativo para Gambino, para Monroy e incluso para Veronica Tomassini, cuyas historias suelen acontecer en áreas suburbanas y estar protagonizadas por antihéroes, por gente con un estilo de vida

\footnotetext{
${ }^{14}$ La propia De Stefani reconoce: "La mentalità della mia famiglia era contraria alla figura dell'artista: l'artista era visto come persona demoniaca destinata ad essere sepolta in terra sconsacrata" (La Monaca 2012) -el libro carece de paginación en el formato electrónico-.
} 
desechable ${ }^{15}$. En el caso de Gambino y Monroy, llama la atención que, pese a hacer alusión al mismo aspecto, ambas lo afrontan desde perspectivas contrapuestas: la primera aborda la partida de los sicilianos al extranjero, mientras que la segunda analiza sobre todo la llegada de inmigrantes a Sicilia, un emplazamiento que, más que ayudarles, los termina aturdiendo todavía más ${ }^{16}$.

Aunque la fascinación por ese azorado universo isleño y por sus enigmas también se constata en los libros publicados por Clara Serretta ${ }^{17}$, queda vivamente de manifiesto en los relatos de Marinella Fiume, una mujer que ha sabido trasladar a sus escritos su bagaje profesional y académico. Esta licenciada en Letras (especialidad en Lenguas Clásicas) no solo muestra su interés por el medio ambiente y por el feminismo ${ }^{18}$, sino, además, por las leyendas -sobre todo grecorromanas, rasgo que comparte con Marilena Monti ${ }^{19}$ - vinculadas a su tierra de origen, una característica que comparte con otras autoras. Simonetta Agnello Hornby y Simona Lo Iacono, por ejemplo, dejan constancia en sus obras de su pasión por el mundo del derecho ${ }^{20}$. Ambas letradas aúnan con

\footnotetext{
${ }^{15}$ Esta característica ya queda retratada en su primera novela: Sangue di cane (2010), un libro en el que volcó su esencia siciliana y la combinó con su interés por el mundo eslavo. La protagonista de esta historia, una mujer siracusana, acabaría, de hecho, enamorándose de un hombre polaco, un varón violento y sin medios que había dejado atrás una cruenta historia de vida.

${ }^{16}$ Así pues, no sorprende que, través de sus escritos, Monroy logre dar cuenta de las singularidades de una tierra enigmática y, en ocasiones, desalmada, patente en fenómenos tan inherentes a este territorio como la emigración. El duro retrato del abandono de la isla -la propia Monroy colaboró con la asociación humanitaria "Emergency" y dio voz a los terribles sufrimientos experimentados por los niños de Lampedusa- constituye, de hecho, uno de los principales ejes temáticos de esta literata: Ragazzo di razza incerta (2013), Dido: operetta pop (2015) y Storia di Giulietta (2018) así lo atestiguan.

${ }^{17}$ Volcada en su carrera de traductora y literata, Clara Serretta plasma el ánima de su amada Sicilia en sus obras. Lo corroboran Forse non tutti sanno che in Sicilia... Curiosità, storie inedite, misteri, aneddoti storici e luoghi sconosciuti di un'isola dalla cultura millenaria (2015) y Alla scoperta dei segreti perduti della Sicilia: itinerari per scoprire nuovi scorci, leggende, aneddoti e tradizioni (2016).

${ }^{18}$ Con respecto al rol social femenino, han de destacarse los siguientes títulos: La vita è infinita (2000), Siciliane (2006), Feudo del mare. La stagione delle donne (2010), Aforismi per le donne toste. Scovati capovolti creati (2012), Di madre in figlia. Vita di una guaritrice di campagna (2014), La sposa nel cuore. Rosaria Nestorini (1926-2010) (2015) y La bolgia delle eretiche (2017).

19 A este respecto, cabe señalar cómo Marilena ha sabido retratar el mundo clásico en su producción literaria. Sus obras Aquila (2001) y La vela, la tela. Lettere inedite tra Ulisse e Penelope (2004) son un claro ejemplo.

${ }^{20}$ Licenciada en Jurisprudencia por la Universidad de Palermo, Simonetta Agnello Hornby abrió en Brixton el despacho de abogados "Hornby\&Levy", especializado en Derecho de familia y del menor.
} 
maestría su interés por las leyes con su afición escritural, un ámbito en el que las mujeres, los marginados y los niños cobran especial protagonismo.

No obstante, las escritoras que se han dedicado en mayor medida al público infantil resultan ser Nadia Terranova -cuya producción se encuentra en su mayoría en la red $^{21}$ - y Lina Maria Ugolini, dos mujeres muy similares en cuanto al tipo de destinatario para el que escriben, pero muy distintas con respecto al contenido. A diferencia de la primera, Ugolini ha sido capaz de integrar en sus libros su talento como musicóloga: sus fábulas y obras de teatro breve, de hecho, atestiguan su afán por establecer una relación entre el texto y la música del siglo Xx, un objetivo al que se suma su pretensión de estudiar el lenguaje cómico de los interludios del siglo XVIII italiano a través de sus ensayos creativos ${ }^{22}$.

Emma Dante, por su parte, representa una vía intermedia entre las literatas que han volcado sus saberes profesionales en sus historias infantiles y aquellas que han reflejado con acierto la realidad de la sociedad más marginal: sirviéndose de una poética dotada de ciertos toques de humor y basada en la tensión y en la locura, buena parte de los textos de esta especialista teatral está dedicada a los niños, un tipo de lector al que atiende con gusto pero en el que no centra toda su atención. Así pues, no es de extrañar que sus narraciones incluso afronten temas mucho más reflexivos y reivindicativos, destinados a remover las conciencias de un sector más adulto ${ }^{23}$. Acerca de su obra, D. Cannova apunta:

Simona Lo Iacono, en cambio, trabaja como jueza en el tribunal de Catania y como voluntaria en la cárcel de Brucoli (Sicilia), donde imparte cursos de literatura, escritura y teatro.

${ }^{21}$ Véase: https://nadiaterranova.com [22 de marzo de 2019].

${ }^{22}$ En relación con este propósito, consúltese: http://www.linamariaugolini.it [22 de marzo de 2019].

${ }^{23}$ Entre sus obras principales se encuentran Carnezzeria. Trilogia della famiglia siciliana (2007), La favola del pesce cambiato (2007), Via Castellana Bandiera (2008), Anastasia, Genoveffa e Cenerentola (2011), La trilogia degli occhiali. Acquasanta, il castello della Zisa e Ballarini (2011), Gli alti e bassi di 
Todavía [se puede] rastrear en la nueva dramaturgia italiana de comienzos del tercer milenio una corriente meridional particularmente activa, definida por muchos como la vanguardia del renacimiento dramatúrgico italiano. Emma Dante, [...] además de otros tantos autores que se encuentran también en el novísimo Centro per la Drammaturgia siciliana, son algunos, entre muchos, de los nombres más conocidos de un fenómeno que exhibe a las regiones del sur de Italia como protagonistas de un movimiento de renacimiento teatral que encuentra en las particulares condiciones económicas, sociales y culturales de esta parte del país un depósito casi inagotable de temas, problemáticas y personajes "en busca de autor". Por alguna formidable alquimia, en estos territorios periféricos $[\ldots]$ se crea un humus particularmente adecuado para el desarrollo de formas "autóctonas" de discurso cultural y artístico que, precisamente, en las idiosincrasias locales encuentran savia para alimentar la creatividad individual. (Cannova 2011: 11-12)

Partiendo de esta idea, Gambino ha reflejado en su producción la importancia de comprender los malos hábitos adquiridos por inercia, motivo por el que ha procurado a través de sus escritos adentrarse en el universo del autodescubrimiento y de la felicidad humana ${ }^{24}$. Entre esas malas prácticas, sobresaldría la que históricamente ha condenado a las mujeres a una posición de inferioridad en relación con los hombres, de ahí que Clelia Lombardo estime oportuno convertir en protagonistas de sus relatos a las féminas olvidadas y valientes que consiguieron reapropiarse con mano firme de su libertad y conocerse mejor a sí mismas ${ }^{25}$. Para ello fue imprescindible la sororidad, un auxilio del que Silvana Grasso ha sabido, asimismo, dar buena cuenta en sus obras ${ }^{26}$.

Por último, el sector culinario ocupa una posición prominente en las obras de las escritoras referidas. Simonetta Agnello Hornby y Clara Serretta han publicado varios

Blancaneve (2013), Io, Nessuno e Polifemo. Intervista impossibile (2014), Le principesse di Emma (2014), Le sorelle Macaluso. Liturgia familiare (2016) y Odissea A/R. Viaggio in due movimenti (2016).

24 Destáquense títulos como Le cattivi abitudini (2010), Ma tu sei felice? Un'indagine sulla felicità (2013) o La perdonanza (2017).

${ }^{25}$ Defensora de la paridad de género, Clelia Lombardo da voz en su texto teatral Ecuba e le altre (2013) a todas esas mujeres que, hartas de sus lóbregas vidas, se percataban de cómo los hombres eran los responsables de una intolerancia que afectaba al conjunto de la población. Pero el interés de esta autora por el teatro y por el activismo feminista no se reduce únicamente a este relato. Prueba de ello son sus obras Trilogia, Labirinti, Il sogno di Maria, Fughe y Andromaca.

${ }^{26} \mathrm{Su}$ interés por temas tan vinculados a Sicilia como la realidad y el misticismo isleños, el rol secundario de las mujeres y el apoyo mutuo femenino queda reflejado en sus libros Nebbie di ddraunàra (1993), 7 uomini 7. Peripezie di una vedova (2006), Pazza è la luna (2007) e Il cuore a destra (2014). 
libros directamente relacionados con la gastronomía, ámbito en el que se incluyen títulos como Un filo d'olio (2011); La cucina del buon gusto (2012); La pecora di Pasqua (2012); Il pranzo di Mosè (2014); Racconti dalla cucina. Quando le storie si fanno golose (2015); Centrifughe, estrati e succhi rigeneranti (2016); Centrifughe, estratti e succhi verdi (2016); Estratti, centrifughe e succhi per vivere cent'anni (2017); y Estratti, centrifughe e smoothies con i superfood (2018). Sin embargo, la fascinación de Agnello y Serretta por este arte es minoritaria en comparación con Giuseppina Torregrossa. Amante de las ambrosías de Sicilia, esta última escritora ha hecho de la comida el núcleo basilar de la mayoría de sus tramas ${ }^{27}$, un elemento sobre el que pivotan las relaciones familiares, las injusticias sociales, la opresión patriarcal o la lucha feminista. En relación con este aspecto, A. Boemi no duda en afirmar cuanto sigue:

La collocazione al centro del bacino del Mediterraneo ha donato all'isola di Sicilia un clima particolarmente mite che ne fa uno dei luoghi più adatti alla vita dell'essere umano. La terra fertilissima, fluviale e vulcanica, il vento, la piovosità, il sole, il mare che la circonda tutta hanno reso la Sicilia una terra ambita, la cui conquista significava, per i diversi popoli che l'hanno abitata, agi e ricchezza. L'evoluzione degli usi alimentari in Sicilia è stata condizionata da tale vantaggio climatico, che ha favorito e favorisce tuttora la crescita di piante tipiche della fascia mediterranea $[\ldots]$ e che ha anche permesso che altre specie vegetali ed animali non autoctone si adattassero con successo alle nuove condizioni [...] Altra condizione che ha prodotto in Sicilia quella che oggi, a detta di illustri esperti [...], è la seconda cucina del mondo per varietà e gusto, seconda solo all'immensa esperienza gastronomica cinese, è la storia di Sicilia, ovvero il contributo fornito da vari popoli con usi alimentari differenti che, sposati a quelli locali, hanno prodotto esiti a dir poco eccezionali. (Boemi 2014: 16-17)

Consciente de esta realidad, Torregrossa ha tenido la innovadora habilidad de incluir en sus relatos todos los rasgos identificativos de su territorio sin renunciar a su tradicional ritual alimenticio. Ha conseguido retratar la esencia de la "sicilianidad" sin dejar flecos pendientes. Y si bien, al igual que muchas otras autoras, esta literata

\footnotetext{
${ }^{27}$ Basta pensar en títulos como Il conto delle minne (2009), Manna e miele, ferro e fuoco (2011), La miscela segreta di casa Olivares (2013) o Il basilico di Palazzo Galletti (2018) para darse cuenta de la predilección de Torregrossa por el ámbito culinario siciliano.
} 
palermitana ha trasladado a todas sus novelas su fervoroso amor por su abnegada profesión -inclúyase su antiguo oficio de médica, ocupación a la que renunció para consagrarse a la escritura- y por su venerada tierra natal, el mayor logro de Torregrossa ha sido hacer partícipe al lector de la espiritualidad femenina de una isla que, paradójicamente, existe, vive y pervive arraigada con fuerza en el machismo.

\section{Conclusiones}

Atentos a las escritoras sicilianas contemporáneas, este artículo ha permitido contextualizar la literatura isleña actual, evidenciando cómo la mujer siempre ha sido sojuzgada y desplazada a un segundo plano. He ahí la razón por la que muchas sicilianas vieron coartadas sus libertades y pocas fueron capaces de emprender una carrera profesional como escritoras. Aun así, las intelectuales de la isla resistieron al machismo imperante en Sicilia y dejaron constancia de la valía de las mujeres en sus escritos, relatos a los que poco a poco se han ido añadiendo nuevos títulos y nombres de autoras que han encontrado en el ámbito literario de comienzos del siglo XXI aquel sitio del que se las había privado impunemente a lo largo de siglos de subyugación.

El acercamiento a la producción literaria de las escritoras sicilianas más recientes ha puesto de manifiesto cómo la mayoría muestra un gran apego por su tierra de origen pese a no compartir el ideario patriarcal siciliano. Sabedoras de la infravaloración de la que son objeto las mujeres -también patente, tal y como señala Daniela Gambino, en la homofobia-, se atreven a retratar una situación social abusiva a la que no todas se aproximan de la misma manera. Marilena Monti y Livia De Stefani, por ejemplo, 
encarnan en cierto modo a dos féminas antagónicas: Monti no duda en reconocer su amor por el dialecto y la cultura de Sicilia, mientras De Stefani tampoco tiene inconveniente en ser crítica, contundente y directa a la hora de tratar en sus escritos el hermetismo ideológico de la isla, un espacio en el que, por su intención de ejercer de escritora, vio limitada su propia autonomía personal.

Atraídas por la cotidianidad insular, Licia Cardillo Di Prima, Teresa Carpinteri, Laura Di Falco, Maria Rosa Cutrufelli, Silvana Grasso, Daniela Gambino, Beatrice Monroy y Marilena Monti contribuyen a definir la esencia de su región natal desde diferentes ópticas: religiosa, costumbrista, mafiosa, opresiva, conservadora, familiar, mística, gastronómica y artística. Desde el punto de vista histórico, el interés concreto por la emigración resulta manifiesto en las obras de Daniela Gambino, Beatrice Monroy y Veronica Tomassini, cada una de las cuales aborda este fenómeno desde distintas experiencias: mientras que Gambino centra su atención en la marcha de los sicilianos al extranjero, Monroy analiza los pormenores de la llegada de inmigrantes a Sicilia y Tomassini orienta la trama de sus narraciones hacia áreas suburbanas habitadas por antihéroes y gentes con un estilo de vida éticamente reprobable.

En cualquier caso, una de las características que más ha llamado la atención de estas escritoras sicilianas es la atmósfera enigmática de su territorio, un ambiente que se plasma con verdadero acierto en algunos de los libros de Clara Serretta y de Marinella Fiume. Con respecto a esta última literata, cabe resaltar su capacidad para verter en sus publicaciones -en las que incluso saca a la luz su interés por el medio ambiente y por el feminismo- su carrera tanto académica como profesional. De ahí que Fiume haya dado cuenta de su predilección -en consonancia con Marilena Monti- por la Sicilia grecorromana, una fascinación análoga a la que Simonetta Agnello Hornby, Simona Lo 
Iacono y Anna Mallamo sienten por su ámbito laboral. El apego por su trabajo y la afición por la escritura de estas tres literatas les han servido para trasladar su trayectoria vital a sus obras, creaciones donde las mujeres, los marginados y los niños ocupan un lugar destacado, y donde el misticismo y la misoginia sicilianos juegan un papel preponderante.

Por otro lado, la elaboración de relatos dedicados al público infantil es un espacio en el que Nadia Terranova y Lina Maria Ugolini se mueven con suma destreza, aunque, eso sí, cada una con un estilo diverso: Terranova evidencia la fuerte conexión que siente con Sicilia a través de la descripción de Mesina, su ciudad natal, mientras que Ugolini ha volcado en sus historias sus grandes dotes como musicóloga, un talento gracias al cual ha conseguido hacerse un nombre en la esfera artística siciliana, ha sido capaz de relacionar el texto y la música del siglo $\mathrm{XX}$, y ha podido estudiar mediante sus ensayos creativos el lenguaje cómico de los interludios del siglo XVIII italiano.

Por su parte, la especialista teatral Emma Dante, haciendo uso de una poética dotada de ciertos toques de humor y basada en la tensión y en la locura, no solo ha compuesto narraciones destinadas a niños, sino también -aunque con un carácter más reflexivo y reivindicativo- a un público más adulto, un sector que, tal y como Daniela Gambino y Clelia Lombardo plasmaron en sus escritos, desgraciadamente ya había aprendido y automatizado de forma espontánea una serie de comportamientos que fomentaban la subestimación de la mujer. He ahí las razones por las que Gambino se abrazó al universo del autodescubrimiento y la felicidad humana, y Lombardo convirtió en protagonistas de sus relatos a mujeres olvidadas y valientes que se reapropiaron de sí mismas y de su libertad. Ambas autoras habían comprendido que solo así sería posible valorar la injusta humillación de un sexo femenino que, conforme a las advertencias de 
Silvana Grasso, hallaba en el apoyo mutuo (es decir, en la sororidad) su verdadero salvavidas.

No resulta, por tanto, sorprendente que la relegación de las sicilianas al ámbito culinario haya sido habitual, una singularidad a la que caerían rendidas gran parte de las literatas isleñas actuales. A tal respecto, es importante destacar que Clara Serretta y Simonetta Agnello Hornby han publicado varios libros relacionados con este arte insular, si bien ha sido Torregrossa quien en verdad ha transformado la gastronomía en una de sus señas literarias personales.

A diferencia del resto de escritoras presentadas, Giuseppina ha hecho de la comida el elemento basilar de buena parte de sus novelas, un componente por medio del cual ha conseguido estimular las relaciones de sus personajes y ha logrado entretejer tramas con las que hacer aflorar el malestar social, la represión machista y la incesante lucha de las sicilianas. Esta innovadora técnica narrativa, unida al amor que Torregrossa profesa por su tierra natal, contribuye a retratar el alma femenina de la "sicilianidad", un alma enraizada en un entorno patriarcal caracterizado por unas contradicciones que esta autora, aún escasamente estudiada, ha dibujado con acierto en su producción.

\section{Referencias bibliográficas}

Aliberti, Carmelo. 2008. Letteratura siciliana contemporanea. Cosenza: Luigi Pellegrini Editore.

2013. L'altra letteratura siciliana contemporanea. Marsala: La Medusa Editrice. 
Baldaro Verde, Jole y Nappi, Rossella. 2002. Donne nuove. L'universo femminile nel terzo millennio. Milano: FrancoAngeli.

Bellerio, Annalisa. 2007. Donne tra le righe. Quaranta scrittrici, una sola grande passione. Milano: Sperling \& Kupfer.

Boemi, Angelo. 2014. Sul mangiare in Sicilia. Tra storia, derivazione e genialità. Catania: Boemi Editore.

Briganti, Alessandra. 1989. Protagoniste e vittime. Le donne e la scrittura. Milano: ESA.

Calapso, Jole, ed. 1980. Donne ribelli. Un secolo di lote femminili in Sicilia. Palermo: Flaccovio Editore.

Cannova, Donatella, ed. 2011. Tres obras dramáticas: nueva dramaturgia italiana. Villa María: Eduvim.

Casarico, Alessandra y Profeta, Paola. 2011. Donne in attesa. L'Italia delle disparità di genere. Milano: Egea.

Cazalé Bérard, Claude. 2009. Donne tra memoria e scrittura. Roma: Carocci Editore.

Corona, Daniela, ed. 1990. Donne e scrittura. Palermo: La Luna.

Correnti, Santi. 2001. Donne di Sicilia: la storia dell'isola del sole scritta al femminile. Trapani: Coppola Editore.

Cucinotta, Giovanni. 1996. Ieri e oggi. Sicilia: storia, cultura, problemi. Cosenza: Luigi Pellegrini Editore.

D'Amico, Nicola. 2015. Storia della formazione professionale in Italia. Dall'uomo da lavoro al lavoro per l'uomo (pref. di G. De Rita). Milano: FrancoAngeli.

Ferlita, Salvatore. 2004. Altri siciliani: scritti sulla letteratura isolana contemporanea. Palermo: Kalós Edizioni.

González de Sande, Mercedes y García Fernández, José. 2018. Enrichetta Caracciolo: Los misterios del convento napolitano. Sevilla: Benilde Ediciones.

Insana, Jolanda. 2007. Tutte le poesie 1977-2006. Milano: Garzanti.

Iuso, Anna, ed. 1999. Scritture di donne. Uno sguardo europeo. Arezzo: Protagon Editori Toscani.

La Monaca, Donatella. 2012. Scrittrici siciliane del Novecento. Palermo: Flaccovio Editore.

Letizia, Laura. 2008. Donne di Sicilia. Marina di Patti (Messina): Pungitopo. 
Livi, Grazia. 1992. Da una stanza all'altra. Milano: La Tartaruga. 2002. Narrare è un destino. Da Virginia Woolf a Karen Blixen, da Anna Banti a Dolores Prato. Milano: La Tartaruga.

Lo Jacono, Vittorio y Zanda, Carmen. 2013. La Sicilia delle donne (intr. di R. Cedrini). Palermo: Spazio Cultura Edizioni.

Mafai, Simona et al. 1976. Essere donna in Sicilia. Roma: Editori Riuniti.

Pigozzi, Elena y De Petris, Susi, ed. 1998. Atlante della letteratura al femminile: l'anima dell'altra metà del cielo. Colognola ai Colli (Veneto): Demetra.

Pruna, Maria Letizia. 2010. Donne al lavoro. Una rivoluzione incompiuta. Bologna: Il Mulino.

Randazzo, Salvatore Basilio. 1985. Sicilianità: subcultura, tradizioni, ethos $e$ comportamenti, tendenzialità. Palermo: Edi Oftes.

Rasy, Elisabetta. 1984. Le donne e la letteratura. Roma: Editori Riuniti.

Resta, Gianvito. 1984. "Prolusione". Letteratura siciliana al femminile: donne scrittrici e donne personaggio. Ed. Sarah Zappulla Muscarà. Caltanissetta-Roma: Salvatore Sciascia Editore. 5-9.

Savatteri, Gaetano. 2017. Non c’è più la Sicilia di una volta. Roma-Bari: Laterza.

Tommasi, Wanda. 2004. La scrittura del deserto. Malinconia e creatività femminile. Napoli: Liguori Editore.

Woolf, Virginia. 1995. Una stanza tutta per sé (trad., ed. di G. Mistrulli). Rimini: Guaraldi Editore.

2003. Le donne e la scrittura (ed. M. Barret, trad. di A. Bottini). Milano: La Tartaruga.

\section{Webgrafía}

Página web oficial de Nadia Terranova. https://nadiaterranova.com [22 de marzo de 2019].

Página web oficial de Lina Maria Ugolini. http://www.linamariaugolini.it [22 de marzo de 2019]. 